Case Report

\title{
Prenatal Diagnosis of Placental Mesenchymal Dysplasia with 46, X, Isochromosome Xq/45, X Mosaicism
}

\author{
Chin-Chieh Hsu ${ }^{1}$, Chien-Hong Lee ${ }^{2}$, Shuenn-Dyh Chang ${ }^{1}$, Tsang-Ming Ko ${ }^{3}$, Shir-Hwa Ueng ${ }^{4}$, Yu-Hsiu Chen ${ }^{4}$, \\ Mei-Chia Wang ${ }^{2}$ and Yao-Lung Chang $1, *$ (i)
}

1 Department of Obstetrics and Gynecology, Chang Gung Memorial Hospital, Linkou Medical Center, College of Medicine, Chang Gung University, Taoyuan 33302, Taiwan; b9602047@cgmh.org.tw (C.-C.H.); gene@cgmh.org.tw (S.-D.C.)

2 Department of Laboratory Medicine, Chang Gung Memorial Hospital, Linkou Medical Center, College of Medicine, Chang Gung University, Taoyuan 33302, Taiwan; 1i5592@cgmh.org.tw (C.-H.L.); ottermika@gmail.com (M.-C.W.)

3 Genephile Bioscience Laboratory, Ko's Obstetrics and Gynecology, Taipei 10050, Taiwan; tsangming@live.com

4 Department of Pathology, Chang Gung Memorial Hospital, Linkou Medical Center, College of Medicine, Chang Gung University, Taoyuan 33302, Taiwan; shu922@cgmh.org.tw (S.-H.U.); cyh0914@cgmh.org.tw (Y.-H.C.)

* Correspondence: j12054@cgmh.org.tw

Citation: Hsu, C.-C.; Lee, C.-H.; Chang, S.-D.; Ko, T.-M.; Ueng, S.-H.; Chen, Y.-H.; Wang, M.-C.; Chang, Y.-L. Prenatal Diagnosis of Placental Mesenchymal Dysplasia with 46, X, Isochromosome Xq/45, X Mosaicism. Genes 2022, 13, 245. https://doi.org/ 10.3390 /genes 13020245

Academic Editor: Rossella Tomaiuolo

Received: 16 December 2021

Accepted: 24 January 2022

Published: 27 January 2022

Publisher's Note: MDPI stays neutral with regard to jurisdictional claims in published maps and institutional affiliations.

Copyright: (C) 2022 by the authors. Licensee MDPI, Basel, Switzerland. This article is an open access article distributed under the terms and conditions of the Creative Commons Attribution (CC BY) license (https:// creativecommons.org/licenses/by/ $4.0 /)$.

\begin{abstract}
Placental mesenchymal dysplasia is an uncommon vascular anomaly of the placenta with characteristics of placentomegaly and multicystic appearance and with or without association with fetal chromosomal anomaly. We present a unique placental mesenchymal dysplasia patient with amniotic fluid karyotyping as 46, X, iso(X) (q10). Detailed molecular testing of the amniotic fluid, fetal cord blood, non-dysplastic placenta and dysplastic placenta was conducted after termination of pregnancy, from which we proved biparental/androgenetic $(46, X, i(X)(q 10) / 45, X)$ mosaicism in different gestational tissues. A high portion of androgenetic cells in dysplastic placenta (74.2\%) and near $100 \%$ of biparental cells in the fetus's blood and amniotic fluid were revealed. Delicate mosaic analyses were performed, and possible pathogenesis and embryogenesis of this case were drawn up.
\end{abstract}

Keywords: placental mesenchymal dysplasia; biparental/androgenetic mosaicism; isochromosome X; $45 \mathrm{X}$; array CGH; microsatellite; short tandem repeats; karyotype

\section{Introduction}

Placental mesenchymal dysplasia (PMD), also known as "pseudo hydatidiform mole", is characterized by a large placenta with grape-like, multicystic change [1]. This rare disease is often misdiagnosed as a partial molar pregnancy due to similar appearance under ultrasonography. However, the karyotype of a partial mole is usually triploid with multiple anomalies of the fetus. In contrast, PMD can show a diploid karyotype of fetus and structurally normal fetus. Distinguishing between the two etiologies mentioned above is important because a normal fetus could be born alive from a pregnancy with PMD, though $33 \%$ of them will have intra-uterine growth restriction, and $52 \%$ will have preterm birth. Peripartum complications also occur in mothers who have PMD, including gestational hypertension and pre-eclampsia [2].

Abnormal genetic composition of the fetus is seen in one-third of cases with PMD, including aneuploidy, uniparental disomy and Beckwith-Wiedemann syndrome. Additionally, by using molecular techniques, biparental/androgenetic mosaicism is ascertained in dysplastic placenta in some literature, which results from aberrant embryogenesis of a single zygote [3]. Here, we present a patient who was diagnosed as PMD and was carrying an euploid female fetus with a chromosomal anomaly detected by amniocentesis as $46, X, i(X)(q 10)$. The dysplastic placenta showed high level of androgenetic $45, X$ 
mosaicism. Serial molecular analyses, including cytogenetic studies, array comparative genomic hybridization (array CGH) and short tandem repeat (STR) analysis were undertaken in order to demonstrate gradient change of the two mosaic cell lines and the possible route of pathogenesis.

\section{Materials and Methods}

\subsection{Patient}

Here, we present a 35-year-old female, who was pregnant at 15 weeks of gestation when she first visited our clinic. This is her second pregnancy, and her previous pregnancy ended with spontaneous abortion at early gestation. She was pregnant after using clomiphene. At 15 weeks of gestation, ultrasonography showed multicystic changes in the placenta with a viable, normally structured fetus (Figure 1a). The placental cysts ranged in size from $0.5-1.0 \mathrm{~cm}$. Adequate fetal growth and normal amniotic fluid amount were seen. Biochemistry study from maternal serum revealed $\beta-\mathrm{hCG}$ as $58,247 \mathrm{mIU} / \mathrm{mL}$ and an elevated $\alpha$-fetoprotein of $149.3 \mathrm{ng} / \mathrm{mL}$ (which was 3.73 multiple of median (MoM)) [4]. Based on ultrasound findings and an elevated maternal serum $\alpha$-fetoprotein, PMD was suspected, and detailed prenatal genetic testing was suggested. Amniocentesis was performed at 17 weeks, which revealed the karyotype as $46, X$, isochromosome Xq10. Array $\mathrm{CGH}$ confirmed the copy number variation as Xp deletion and Xq duplication. Level 2 ultrasound showed normal fetal structure and an enlarged placenta containing two parts: dysplastic and non-dysplastic parts (Figure 1b). Low vascularity was detected in the dysplastic placenta via Doppler scan and 3D reconstruction (Figure 1c,d). Genetic counseling was given, and the mother decided to terminate the pregnancy due to an isochromosome of the long arm of chromosome X, a Turner syndrome variant. An abortus with its dysmorphic placenta was delivered smoothly. The fetus's general appearance was normal without omphalocele, macroglossia or appearing macrosomic.

The gestational tissue, including the amniotic fluid from prenatal amniocentesis, cord blood and placental biopsy after delivery, were collected and sent for further cytogenetic and molecular evaluation, and the remaining placenta was fixed for histopathological examination.

\subsection{Cytogenetic Studies: Cell Culture and Karyotyping}

For amniotic fluid cells and placenta tissue, long-term culture ( $>7$ days) was applied with Chang medium T101-019 (Irvine Scientific, Santa Ana, CA, USA). For cord blood, Phytohemagglutinin Assay (PHA) was used to stimulate mitosis of peripheral lymphocytes under short-term culture (2-3 days) with Chang medium. G-banding technique was performed after cell fixation, and 450-band resolution was acquired. For every sample, 20 cells during metaphase were counted, which were derived from at least 2 isolated colonies.

\subsection{DNA Extraction}

DNA extractions on cord blood, amniotic fluid and placental tissue were performed for studies by array CGH, a short tandem repeat analysis and methylation-specific multiplex ligation-dependent probe amplification (MS-MLPA). QIAamp DNA mini kit was used according to the manufacturers' guide.

\subsection{Array Comparative Genomic Hybridization (Array CGH)}

We used the SurePrint G3 ISCA V2 Human CGH 8X60K Array Kit (Agilent Technologies, Santa Clara, CA, USA) to identify genomic coping number variation (CNV) in testing sample. After DNA extraction, fluorescent labeling was performed with a SureTag DNA Labeling Kit (Agilent Technology). Cy5-dUTP was applied on the examined tissues, and Cy3-dUTP was applied on reference human genomic DNA (Male-Promega G147A, Female-Promega G152A). After hybridization with microarray chip, the result was read via a SureScan Microarray scanner (Agilent Technology) and analyzed with Agilent CytoGenomics, edition 2.7.8.0. A mosaic mode analyzer was used on dysplastic and non-dysplastic 
placenta due to known androgenetic/biparental mosaicism. The ratio of two mosaic cell lines was calculated using mean log ratio by experienced technologist (Lee, Chien-Hong).
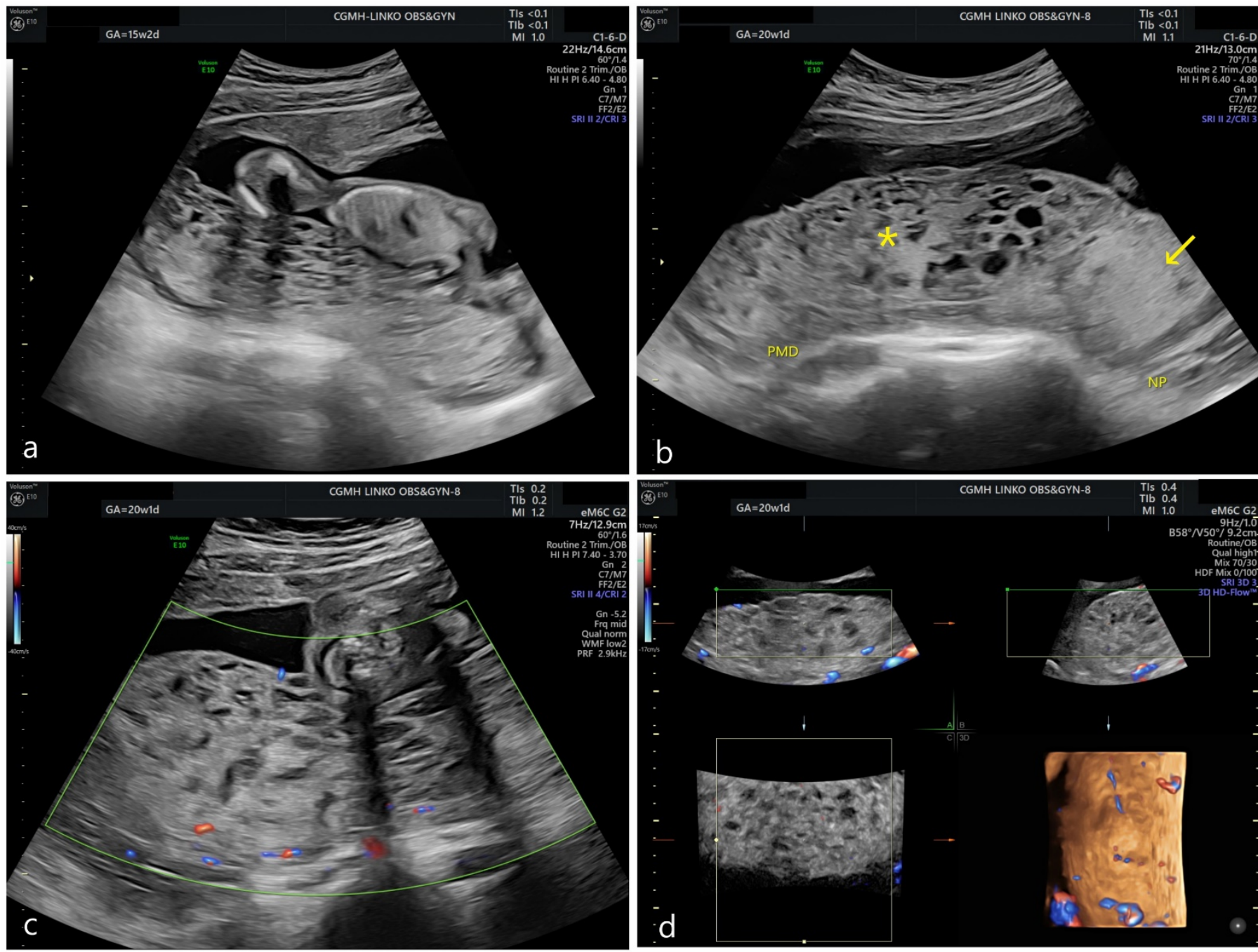

Figure 1. Prenatal ultrasonography. (a) At 15 weeks of gestation, multicystic change of placenta could be seen. (b) At 20 weeks of gestation, an enlarged dysplastic portion of the placenta was detected. (star *: dysplastic placenta; arrow: non-dysplastic placenta; PMD: placental mesenchymal dysplasia; NP: normal placenta) (c) Under Doppler scan, the dysplastic placenta showed low vascularity. (d) Under 3D reconstruction, the dysplastic placenta remained avascular.

\subsection{Histology}

Between 1 and 4 representative sections were selected from the dysplastic and the nondysplastic part of the placenta. After formalin-fixed and paraffin-embedded tissue section, P57 immunohistochemistry was performed using Anti-p53 Antibody (clone DO-7, Leica biosystems, Buffalo Grove, IL, USA). The nuclear staining of stromal cell was reviewed by two pathologists.

\subsection{Short Tandem Repeat (STR) Analysis}

We used GlobalFiler Express PCR Amplification kit (Thermo Fisher, Waltham, MA, USA), GenePhile X-Plex PCR Amplification Kit (GenePhile Bioscience Co., Ltd., Taipei City, Taiwan) and 3500 DX Genetic Analyzer (Thermo Fisher) to count different tetranucleotide repeats and distinguish maternal or paternal origin of the examined alleles. In autosomal chromosomes, GlobalFiler Express PCR Amplification kit (Thermo Fisher) designs 21 STR loci using (D3S1358, vWA, D16S539, CSF1PO, TPOX, D8S1179, D21S11, D18S51, D2S441, 
D19S433, TH01, FGA, D22S1045, D5S818, D13S317, D7S820, SE33, D10S1248, D1S1656, D12S391, D2S1338) as markers, while 13 loci (DXS6807, DXS8378, DSX9902, DXS7132, DXS9898, DXS6809, DXS6789, DXS7424, DXS101, GATA172D05, HPRTB, DXS8377, DXS7423) on X chromosome were applied by GenePhile X-Plex PCR Amplification Kit (GenePhile Bioscience Co., Ltd.) [5]. The data were managed with Genescan (software), and the biparental-to-androgenetic ratio was calculated using the under-curve area by experienced technologist (Lee, Chien-Hong).

\subsection{Methylation-Specific Multiplex Ligation-Dependent Probe Amplification (MS-MLPA)}

To enable a diagnosis of the Beckwith-Weidemann syndrome, MS-MLPA was performed with SALSA MS-MLPA Probemix specific kit ME030-C3 BWS/RSS to identify abnormal epigenetic methylation or coping number variation (CNV) in chromosome 11p15 BWS/RSS region, which contains multiple probes targeting two imprinted domains, IC1(H19DMR) and IC2(KvDMR), and two related genes, the H19 gene and the KCNQ1OT1 gene.

\subsection{Mosaic Analysis}

Mean log ratio from array CGH and area under curve (AUC) from STR were used for mosaic analysis. In array $\mathrm{CGH}$, assuming the mosaic phenomenon consisted of two genotypes, 46, X, i(X) (q10) and 45, X, we set $\delta$ for the portion of $46, X, i(X)(q 10)$ and $N$ for ploidy of chromosome Xq. The formula was designed as

$$
\begin{gathered}
\text { mean } \log \text { ratio }=-\log _{2} \frac{\mathbf{N}}{\mathbf{2}} \\
N=3 \delta+(1-\delta)=2 \delta+1 \\
\delta=\frac{\mathrm{N}-1}{2} \times 100 \%
\end{gathered}
$$

In STR analysis, markers of autosomal chromosomes are used to evaluate biparental/ androgenetic mosaicism, and markers of $X$ chromosome are applied to estimate $46, X$, isochromosome $\mathrm{Xq} / 45, \mathrm{X}$ mosaicism. By using the area under curve (AUC), the ratio of parental signals could be transferred to mosaic rate. From markers of autosomal chromosomes, taking $\mathrm{P}$ for parental signals, $\mathrm{M}$ for maternal signals and the portion of biparental cells $(x)$ could be calculated as follows:

$$
\begin{aligned}
& \frac{\mathrm{P}}{\mathrm{M}}=\frac{x+2(1-x)}{x} \\
& x=\frac{2 \mathrm{M}}{\mathrm{P}+\mathrm{M}} \times 100 \%
\end{aligned}
$$

From markers of $\mathrm{X}$ chromosome, taking $\mathrm{P}$ for parental signals, $\mathrm{M}$ for maternal signals and $y$ as the portion of $46, X, i(X)(q 10)$, the AUC ratio could be presented as

$$
\begin{gathered}
\frac{\mathrm{P}}{\mathrm{M}}=\frac{y+(1-y)}{2 y} \\
\mathrm{y}=\frac{\mathrm{M}}{2 \mathrm{P}} \times 100 \%
\end{gathered}
$$

\section{Results}

After delivery, the placenta grossly presented with two kinds of morphology: a dysplastic part with grape-like, multicystic change, and a non-dysplastic part with near-normal appearance. The ratio of the area over the dysplastic and the non-dysplastic part was about $7: 1$, and the umbilical cord was centrally inserted and divided symmetrically to the whole placenta (Figure 2). Under the hematoxylin-eosin ( $\mathrm{H}$ and $\mathrm{E}$ ) stain, the dysplastic 
placenta showed abnormally enlarged villi with cavernous cysts and loose myxoid stroma (Figure 3a). Abnormal chorangiomatosis-like vascular proliferation within some villi was seen (Figure 3b). There was no significant trophoblastic proliferation, which was compatible with the diagnosis of placenta mesenchymal dysplasia rather than molar pregnancy. Immunohistochemistry stain for p57 showed a complete loss of expression of the stromal cells in the dysplastic part of the placenta, indicating a loss of expression from maternal genome (Figure 3c,d) [6].

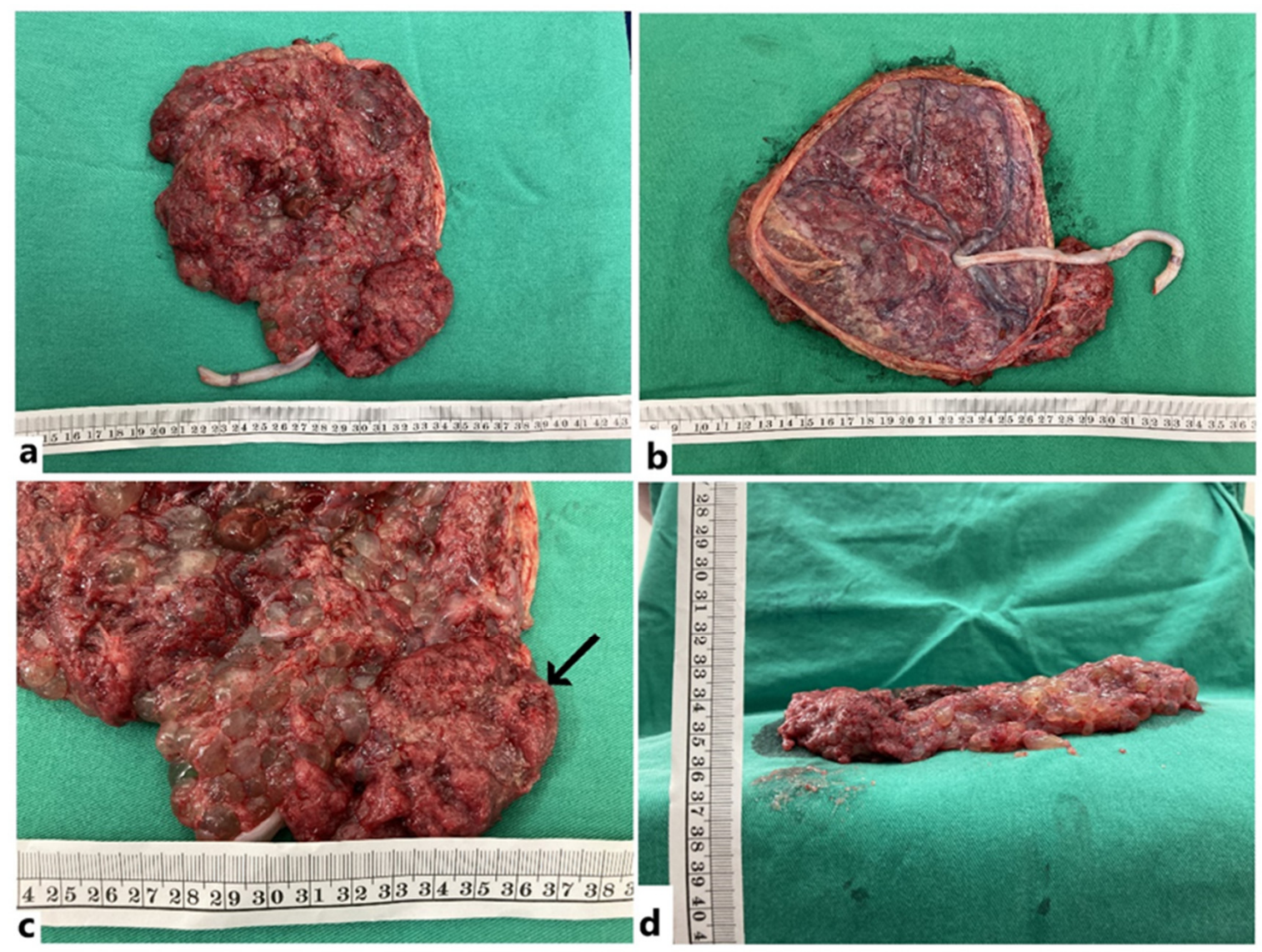

Figure 2. Gross appearance of the placenta and the fetus. (a) Maternal side; (b) Fetal side with centrally inserted umbilical cord; (c) Small portion of non-dysplastic placenta (arrow); (d) Lateral view of placenta.

Karyotype of cord blood, non-dysplastic placenta and dysplastic placenta showed diverse results. Cord blood was karyotyped as 46, X, i(X) (q10), but non-dysplastic and dysplastic placenta showed $45, X$ without mosaicism. These two kinds of genotype were assumed to be the main composition of the gestational products, including the placenta, amniotic fluid and fetus, in the following analysis.

An array CGH of uncultured cells from cord blood, non-dysplastic and dysplastic placenta revealed inconsistent copy number variation of $X$ chromosome. By calculation, portions of 46, X, i(X) (q10) in cord blood, non-dysplastic and dysplastic placenta were $98.69 \%, 69.01 \%, 22.43 \%$, and portions of 45 , $X$ were $1.31 \%, 30.99 \%, 77.57 \%$, respectively. Amniotic fluid from prenatal amniocentesis showed totally with 46, X, i(X) (q10) (Table 1).

The analysis of short tandem repeats (STR) helps to distinguish parental origin of the genome, as shown in Figure 4. Markers of autosomal chromosomes are located in chromosomes 1 to 21, and markers of sex chromosomes are located on both the $\mathrm{p}$ arm and $\mathrm{q}$ arm of $X$ chromosome. Through a comparison with parents' STR number, the result showed that all products of conception shared the same allelic composition, indicating the single zygotic origin. However, the different ratio of paternal and maternal signals 
from specimens implied possible androgenetic/biparental mosaicism. Particularly in the dysplastic placenta, paternal signals were significantly greater than maternal signals, suggesting an androgenetic-dominant composition. In Table 2, STR markers on autosomes were using to demonstrate androgenetic/biparental mosaic ratio by calculating the area under curve (AUC). The cord blood, amniotic fluid and non-dysplastic placenta showed nearly $100 \%$ biparental cells. However, the portion of biparental cells in the dysplastic placenta was only $25.80 \%$.

In addition, the absence of markers on the $\mathrm{p}$ arm of maternal $\mathrm{X}$ chromosome reveals the maternal origin of isochromosome $\mathrm{Xq}$ (Table 3). The proportion of isochromosome $\mathrm{Xq}$ in the non-dysplastic placenta and the dysplastic placenta were $65.25 \%$ and $8.16 \%$, respectively. On the other hand, the amniotic fluid and cord blood showed nearly totally 46, X, isochromosome Xq. The result was compatible with array CGH (Table 1).
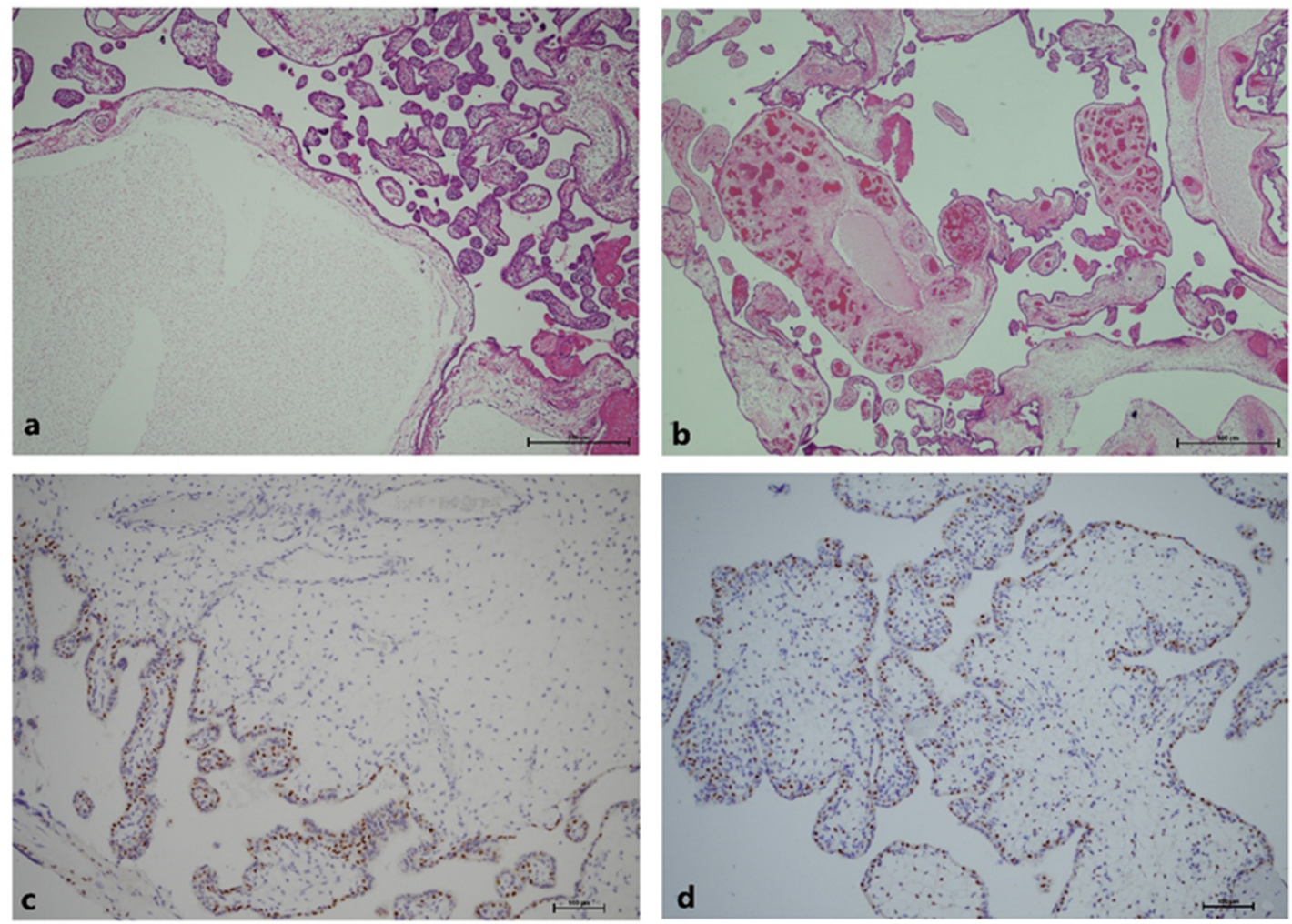

Figure 3. Histology of dysplastic placenta. (a) Dysplastic villi under hematoxylin-eosin stain. An edematous, dysplastic villi was surrounded by some normal villi, showing a mosaic pattern of histological appearance; (b) Chorangiosis in dysplastic villi; (c) P57 stain of dysplastic villi. No nuclear uptake in stroma is seen, indicating no expression of maternal genome; (d) P57 stain of normal villi. Nuclear stain in stroma is seen.

Table 1. Mosaic analysis of array CGH.

\begin{tabular}{ccccc}
\hline & Amniotic Fluid & Cord Blood & $\begin{array}{c}\text { Non-Dysplastic } \\
\text { Placenta }\end{array}$ & $\begin{array}{c}\text { Dysplastic } \\
\text { Placenta }\end{array}$ \\
\hline Mean log ratio & 0.591397 & 0.572296 & 0.251074 & -0.46537 \\
\hline $\mathrm{N} / 2$ & 1.506705 & 1.486888 & 1.190093 & 0.724287 \\
\hline $\mathrm{N}$ & 3.01341 & 2.973776 & 2.380186 & 1.448574 \\
\hline$\delta$ & $100.67 \%$ & $98.69 \%$ & $69.01 \%$ & $22.43 \%$ \\
\hline $1-\delta$ & $-0.67 \%$ & $1.31 \%$ & $30.99 \%$ & $77.57 \%$
\end{tabular}

Mean $\log$ ratio: $-\log 2(\mathrm{~N} / 2)$. N: ploidy of chromosome Xq. $\delta$ : the portion of $46, X, i(X)(q 10) .1-\delta$ : the portion of 45 , X. 


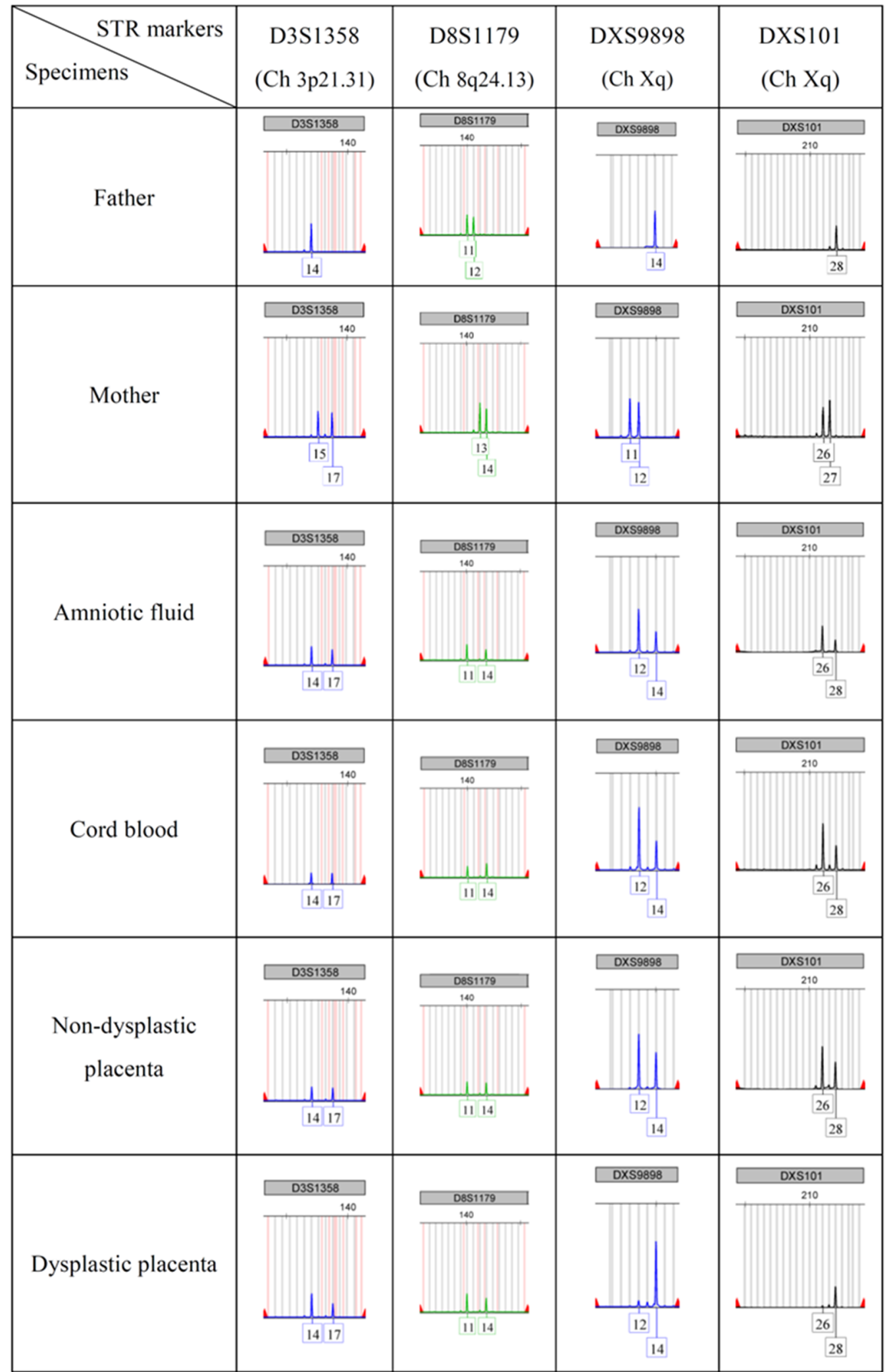

Figure 4. Short tandem repeat (STR) of blood of father, blood of mother, amniotic fluid, cord blood, non-dysplastic placenta and dysplastic placenta. All products of conception shared the same allelic signal. 
Table 2. Mosaic analysis of androgenetic/biparental cell lines using STR markers from autosomal chromosomes.

\begin{tabular}{|c|c|c|c|c|c|c|c|c|c|c|c|c|c|}
\hline \multirow[b]{2}{*}{$\begin{array}{c}\text { STR } \\
\text { Markers }\end{array}$} & \multirow[b]{2}{*}{$\begin{array}{c}\text { Chromosomal } \\
\text { Location }\end{array}$} & \multicolumn{3}{|c|}{ Amniotic Fluid } & \multicolumn{3}{|c|}{ Cord Blood } & \multicolumn{3}{|c|}{ Non-Dysplastic Placenta } & \multicolumn{3}{|c|}{ Dysplastic Placenta } \\
\hline & & $\begin{array}{l}\text { Allele } \\
\text { (P/M) }\end{array}$ & $\begin{array}{l}\text { AUC } \\
(\mathbf{P} / \mathbf{M})\end{array}$ & $\begin{array}{l}\text { Biparental } \\
\text { Rate }\end{array}$ & $\begin{array}{l}\text { Allele } \\
\text { (P/M) }\end{array}$ & $\begin{array}{l}\text { AUC } \\
\text { (P/M) }\end{array}$ & $\begin{array}{c}\text { Biparental } \\
\text { Rate }\end{array}$ & $\begin{array}{l}\text { Allele } \\
\text { (P/M) }\end{array}$ & $\begin{array}{l}\text { AUC } \\
\text { (P/M) }\end{array}$ & $\begin{array}{c}\text { Biparental } \\
\text { Rate }\end{array}$ & $\begin{array}{l}\text { Allele } \\
\text { (P/M) }\end{array}$ & $\begin{array}{l}\text { AUC } \\
(\mathbf{P} / \mathbf{M})\end{array}$ & $\begin{array}{c}\text { Biparental } \\
\text { Rate }\end{array}$ \\
\hline D3S1358 & $3 p 21.31$ & $14 / 17$ & $16,751 / 14,151$ & $91.59 \%$ & $14 / 17$ & $18,393 / 17,785$ & $98.32 \%$ & $14 / 17$ & $15,856 / 14,804$ & $96.57 \%$ & $14 / 17$ & $48,076 / 6164$ & $22.73 \%$ \\
\hline vWA & 12 p13.31 & $17 / 18$ & $27,518 / 24,229$ & $93.64 \%$ & $17 / 18$ & $41,960 / 33,329$ & $88.54 \%$ & $17 / 18$ & $27,448 / 30,097$ & $104.60 \%$ & $17 / 18$ & $66,220 / 11,697$ & $30.02 \%$ \\
\hline D16S539 & $16 q 24.1$ & $9 / 11$ & $32,759 / 36,536$ & $105.45 \%$ & $9 / 11$ & $78,624 / 76,666$ & $98.74 \%$ & $9 / 11$ & $66,014 / 61,394$ & $96.37 \%$ & $9 / 11$ & $120,006 / 11,389$ & $17.34 \%$ \\
\hline D8S1179 & $8 \mathrm{q} 24.13$ & $11 / 14$ & $16,840 / 11,381$ & $80.66 \%$ & $11 / 14$ & $18,445 / 22,999$ & $110.99 \%$ & $11 / 14$ & $18,056 / 16,524$ & $95.57 \%$ & $11 / 14$ & $48,812 / 6321$ & $22.93 \%$ \\
\hline D18S51 & $21 q 11.2-q 21$ & $15 / 16$ & $93,826 / 104,530$ & $105.40 \%$ & $15 / 16$ & $149,449 / 118,218$ & $88.33 \%$ & $15 / 16$ & $102,685 / 98,713$ & $98.03 \%$ & $15 / 16$ & $158,985 / 31,591$ & $33.15 \%$ \\
\hline D2S441 & $18 \mathrm{q} 21.33$ & $11 / 13$ & $39,236 / 27,800$ & $82.94 \%$ & $11 / 13$ & $47,803 / 47,727$ & $99.92 \%$ & $11 / 13$ & $41,749 / 43,326$ & $101.85 \%$ & $11 / 13$ & $86,278 / 14,227$ & $28.31 \%$ \\
\hline TH01 & 2p14 & $9 / 7$ & $42,235 / 53,801$ & $112.04 \%$ & $9 / 7$ & $70,117 / 80,395$ & $106.83 \%$ & $9 / 7$ & $63,412 / 66,071$ & $102.05 \%$ & $9 / 7$ & $109,520 / 18,859$ & $29.38 \%$ \\
\hline FGA & $19 q 12$ & $20 / 23$ & $45,652 / 55,661$ & $109.88 \%$ & $20 / 23$ & $97,451 / 96,252$ & $99.38 \%$ & $20 / 23$ & $69,714 / 83,480$ & $108.99 \%$ & $20 / 23$ & $111,815 / 14,095$ & $22.39 \%$ \\
\hline D5S818 & 11 p15.5 & $9 / 13$ & $36,069 / 24,468$ & $80.84 \%$ & $9 / 13$ & $38,269 / 37,060$ & $98.40 \%$ & $9 / 13$ & $25,995 / 25,416$ & $98.87 \%$ & $9 / 13$ & $78,297 / 10,763$ & $24.17 \%$ \\
\hline D13S317 & $4 q 28$ & $10 / 11$ & $42,997 / 42,240$ & $99.11 \%$ & $10 / 11$ & $70,928 / 54,565$ & $86.96 \%$ & $10 / 11$ & $37,985 / 39,182$ & $101.55 \%$ & $10 / 11$ & $95,033 / 13,547$ & $24.95 \%$ \\
\hline SE33 & $5 q 21-31$ & $27.2 / 18$ & $128,927 / 130,009$ & $100.42 \%$ & $27.2 / 18$ & $213,111 / 210,357$ & $799.35 \%$ & $27.2 / 18$ & $180,405 / 160,701$ & $94.22 \%$ & $27.2 / 18$ & $218,007 / 48,467$ & $36.38 \%$ \\
\hline D10S1248 & $13 q 22-31$ & $14 / 15$ & $18,771 / 14,955$ & $88.69 \%$ & $14 / 15$ & $18,719 / 19,616$ & $102.34 \%$ & $14 / 15$ & $13,096 / 11,450$ & $93.29 \%$ & $14 / 15$ & $49,175 / 3700$ & $14.00 \%$ \\
\hline D12S391 & $6 q 14$ & $23 / 18$ & $40,624 / 42,025$ & $101.70 \%$ & $23 / 18$ & $55,074 / 72,839$ & $113.89 \%$ & $23 / 18$ & $37,519 / 42,589$ & $106.33 \%$ & $23 / 18$ & $98,544 / 16,879$ & $29.25 \%$ \\
\hline \multirow[t]{2}{*}{ D2S1338 } & $10 \mathrm{q} 26.3$ & $19 / 24$ & $67,167 / 65,573$ & $98.80 \%$ & $19 / 24$ & $69,352 / 61,456$ & $93.96 \%$ & $19 / 24$ & $63,880 / 65,648$ & $101.36 \%$ & $19 / 24$ & $159,569 / 23,995$ & $26.14 \%$ \\
\hline & & \multicolumn{2}{|c|}{ Average } & $96.51 \%$ & \multicolumn{2}{|c|}{ Average } & $99.00 \%$ & \multicolumn{2}{|c|}{ Average } & $99.98 \%$ & \multicolumn{2}{|c|}{ Average } & $25.80 \%$ \\
\hline
\end{tabular}

Assume mosaicism with $(46, X, i(X)(q 10)$, biparental) and (45, $X$, androgenetic). biparental rate $=2 \mathrm{M} /(\mathrm{P}+\mathrm{M})$. 
Table 3. Mosaic analysis of 46, X, iso(Xq) and 45, X using STR markers from sex chromosomes.

\begin{tabular}{|c|c|c|c|c|c|c|c|c|c|c|c|c|c|}
\hline \multirow[b]{2}{*}{$\begin{array}{c}\text { STR } \\
\text { Markers }\end{array}$} & \multirow[b]{2}{*}{$\begin{array}{l}\text { Chromo- } \\
\text { Somal } \\
\text { Location }\end{array}$} & \multicolumn{3}{|c|}{ Amniotic Fluid } & \multicolumn{3}{|c|}{ Cord Blood } & \multicolumn{3}{|c|}{ Non-Dysplastic Placenta } & \multicolumn{3}{|c|}{ Dysplastic Placenta } \\
\hline & & $\begin{array}{l}\text { Allele } \\
\text { (P/M) }\end{array}$ & $\begin{array}{l}\text { AUC } \\
\text { (P/M) }\end{array}$ & $\begin{array}{c}46, X \\
\text { isoXq } \\
\text { Rate }\end{array}$ & $\begin{array}{l}\text { Allele } \\
\text { (P/M) }\end{array}$ & $\begin{array}{l}\text { AUC } \\
(\mathbf{P} / \mathrm{M})\end{array}$ & $\begin{array}{c}46, X \\
\text { isoXq } \\
\text { Rate }\end{array}$ & $\begin{array}{l}\text { Allele } \\
\text { (P/M) }\end{array}$ & $\begin{array}{l}\text { AUC } \\
(\mathbf{P} / \mathbf{M})\end{array}$ & $\begin{array}{c}46, X \\
\text { isoXq } \\
\text { Rate }\end{array}$ & $\begin{array}{l}\text { Allele } \\
\text { (P/M) }\end{array}$ & $\begin{array}{l}\text { AUC } \\
\text { (P/M) }\end{array}$ & $\begin{array}{c}46, X, \\
\text { isoXq } \\
\text { Rate }\end{array}$ \\
\hline DXS6807 & $X p$ & $11 / x(15)$ & $12,848 / 0$ & & $11 / x(15)$ & $55,158 / 0$ & & $11 / x(15)$ & $49,061 / 0$ & & $11 / x(15)$ & $97,868 / 0$ & \\
\hline DXS9902 & $\mathrm{Xp}$ & $10 / x(11)$ & $20,816 / 0$ & & $10 / x(11)$ & $47,862 / 0$ & & $10 / x(11)$ & $51,007 / 0$ & & $10 / x(11)$ & $83,574 / 0$ & \\
\hline DXS9898 & $\mathrm{Xq}$ & $14 / 12$ & $15,843 / 32,626$ & $102.97 \%$ & $14 / 12$ & $60,309 / 126,386$ & $104.78 \%$ & $14 / 12$ & $54,856 / 81,814$ & $74.57 \%$ & $14 / 12$ & $112,201 / 11,273$ & $5.02 \%$ \\
\hline DXS8377 & $\mathrm{Xq}$ & $48 / 51$ & $25,374 / 49,396$ & $97.34 \%$ & $48 / 51$ & $65,812 / 134,888$ & $102.48 \%$ & $48 / 51$ & $68,455 / 83,375$ & $60.90 \%$ & $48 / 51$ & $142,370 / 17,128$ & $6.02 \%$ \\
\hline HPRTB & $\mathrm{Xq}$ & $15 / 12$ & $28,132 / 53,429$ & $94.96 \%$ & $15 / 12$ & $62,571 / 149,412$ & $119.39 \%$ & $15 / 12$ & $71,063 / 100,667$ & $70.83 \%$ & $15 / 12$ & $145,559 / 23,456$ & $8.06 \%$ \\
\hline DXS7132 & $\mathrm{Xq}$ & $14 / 15$ & $36,437 / 60,358$ & $82.83 \%$ & $14 / 15$ & $119,612 / 162,804$ & $68.06 \%$ & $14 / 15$ & $88,222 / 112,703$ & $63.87 \%$ & $14 / 15$ & $165,957 / 26,683$ & $8.04 \%$ \\
\hline DXS101 & $\mathrm{Xq}$ & $28 / 26$ & $17,510 / 37,195$ & $106.21 \%$ & $28 / 26$ & $47,461 / 92,150$ & $97.08 \%$ & $28 / 26$ & $41,584 / 65,929$ & $79.27 \%$ & $28 / 26$ & $93,974 / 20,712$ & $11.02 \%$ \\
\hline DXS6789 & $\mathrm{Xq}$ & $15 / 21$ & $69,262 / 142,895$ & $103.16 \%$ & $15 / 21$ & $175,639 / 22,9088$ & $65.22 \%$ & $15 / 21$ & $164,974 / 190,858$ & $57.84 \%$ & $15 / 21$ & $245,485 / 43,987$ & $8.96 \%$ \\
\hline \multirow[t]{2}{*}{ DXS7424 } & $\mathrm{Xq}$ & $15 / 16$ & $18,594 / 25,692$ & $69.09 \%$ & $15 / 16$ & $38,859 / 61,909$ & $79.66 \%$ & $15 / 16$ & $43,348 / 42,893$ & $49.48 \%$ & $15 / 16$ & $66,848 / 13,377$ & $10.01 \%$ \\
\hline & & \multicolumn{2}{|c|}{ Average } & $93.79 \%$ & \multicolumn{2}{|c|}{ Average } & $90.95 \%$ & \multicolumn{2}{|c|}{ Average } & $65.25 \%$ & \multicolumn{2}{|c|}{ Average } & $8.16 \%$ \\
\hline
\end{tabular}

Assume mosaicism with $(46, X, i(X)(q 10))$ and $(45, X) .46, X$, isoXq rate $=M / 2 P$. 
Finally, MS-MLPA of cord blood was performed and showed no abnormal finding. Beckwith-Wiedemann syndrome was then excluded due to normal fetal growth and normal methylation of the BWS/RSS region (Figure 5).

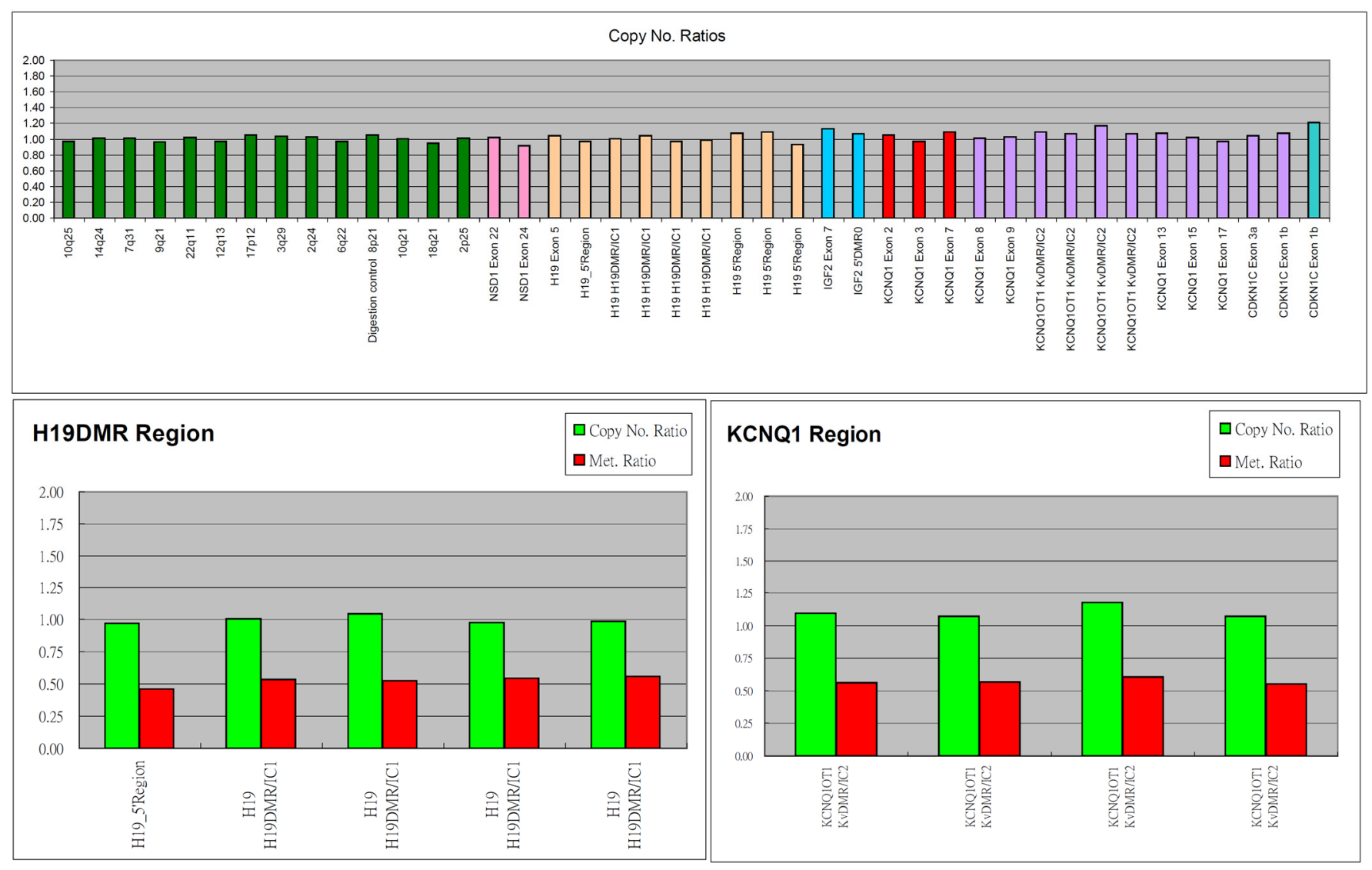

Figure 5. Multiplex ligation-dependent probe amplification (MLPA) of amniotic fluid showed normal methylation of BWS/RSS region.

\section{Discussion}

The incidence of PMD has been estimated as ranging from $0.002 \%$ [7] to $0.02 \%[7,8]$, and the incidence of genotype $46, \mathrm{X}, \mathrm{i}(\mathrm{Xq})$ has been reported as 1 in 25,000 live baby births [9]. It is extremely rare to have these two situations happening coincidentally. From a systemic review [2], 28\% of cases with PMD have abnormal genetic evaluation of the fetus, including chromosomal anomaly, Beckwith-Wiedemann syndrome or uniparental disomy. Chromosomal anomalies have been reported with trisomy 13, Klinefelter's syndrome $(47, \mathrm{XXY}), 69, \mathrm{XXX}$ and mosaicism for trisomy 13. To our knowledge, this is the first case presented with PMD with isochromosome $X q$. The STR analysis of $X$ chromosome shows double dosage of single maternal Xq signals, which hints at the maternal origin of isochromosome $\mathrm{Xq}$ and indicates malsegregation, and the formation of isochromosome occurs at the moment of fertilization, during which meiosis 2 of oocytogenesis completes.

Additionally, this is also the first reported case presenting placental mesenchymal dysplasia with a karyotype of $45, \mathrm{X}$ in the dysplastic placenta. Only one case has been diagnosed where a complete hydatidiform mole displayed a 45, X karyotype [10]. In our case, a distinctly decreased dosage on the whole $\mathrm{X}$ chromosome from array CGH on uncultured dysplastic and non-dysplastic placenta, indicating a larger portion of cells with 45 , X. A karyotype of the dysplastic placenta further confirmed the result. By using STR markers, only $25.8 \%$ cells of dysplastic placenta showed biparental genome (Table 2), and $74.2 \%$ cells presented paternal alleles only. On the other hand, STR markers from X chromosome revealed $65.25 \%$ and $8.16 \%$ cells of non-dysplastic and dysplastic placenta, having 
isochromosome $\mathrm{Xq}$, which means about $34.75 \%$ and $91.84 \%$ cells are karyotyped as 45 , $\mathrm{X}$ in these two parts of the placenta, respectively. Because of the merely $25 \%$ of biparental alleles seen in the dysplastic placenta and the different composition of biparental/androgenetic mosaicism and 46, X, isochromosome Xq/45, X mosaicism, we believe most cells in the dysplastic placenta should be a uniparental $45, X$ (paternal), which may come from a sperm with haploid endoduplication and subsequent failure of replication of $\mathrm{X}$ chromosome (Figure 6).

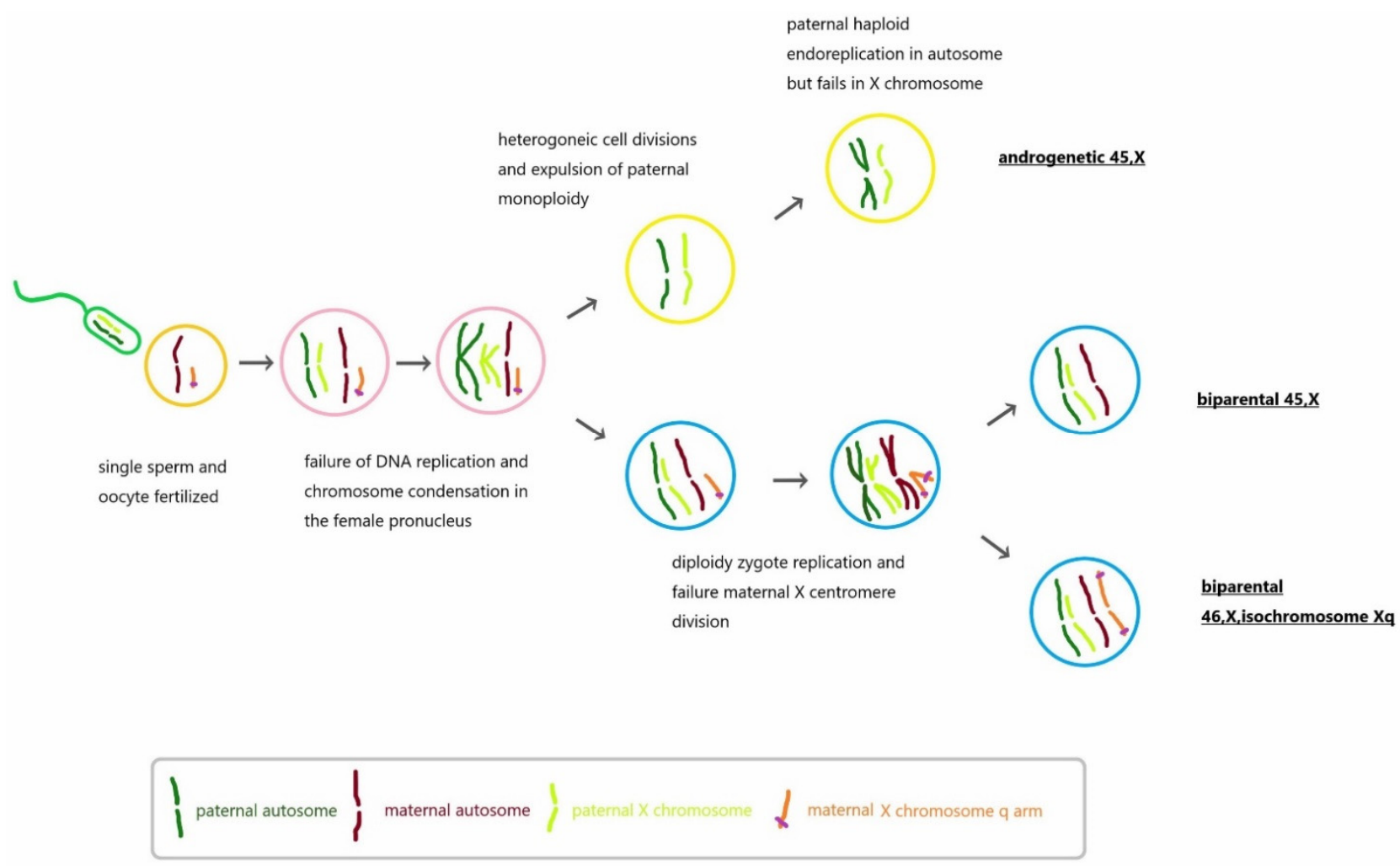

Figure 6. Hypothetical cause of biparental/androgenetic mosaicism and 46, $X$, isochromosome $\mathrm{Xq} / 45, \mathrm{X}$ mosaicism. Single sperm was fertilized with the oocyte carrying 22, Xq. Before the female pronucleus condensed, the paternal genome replicated first and was expelled as distinct cell, which is called heterogeneous cell division. The autosomes of paternal haploid endoreplicated but failed in X chromosome, resulting in the androgenetic 45, X. On the other hand, after heterogeneous cell division, the biparental zygote went through cell replication and mis-division of the centromere on $X$ chromosome, leading to biparental 46, $\mathrm{X}$, isoXq/45, X mosaicism.

It is worth mentioning that the STR analysis showed uniform paternal and maternal signals from somatic chromosome to sex chromosome, suggesting the zygote carries one haploid from a single sperm and the other one from a single oocyte. Under such conditions, the diversity of different genotypes in different gestational tissues should be described as "mosaicism" instead of "chimerism". Androgenetic/biparental mosaicism causing placental mesenchymal dysplasia was first announced by K. A. Kaiser-Rogers in 2006 [3]. In that study, microsatellite markers for chromosome 7, 9, 12, 14, 16, X were applied to check the parental origin of the genome from amniotic fluid, fetus and chorions, and they found an increased proportion of androgenetic cells in chorionic mesenchyme and enlarged chorionic vessels on the placenta, from $80 \%$ to $100 \%$ [3]. In our case, the androgenetic rate is also higher in the dysplastic placenta $(74.20 \%$, androgenetic rate $=1-$ biparental rate, Table 2), and nearly no androgenetic cells are seen in the fetal cord blood by using STR analysis.

According to Aspasia Destouni, et al. [11], several post-zygotic events contribute to chromosomal instability and may lead to mosaicism or mixoploidy. Androgenetic 
mixoploidy, which is defined as cells with only paternally derived genome and different ploidy status, can be seen in human diseases, including PMD, complete hydatidiform mole or partial hydatidiform moles. In their study, they named the phenomenon of spontaneous segregation of whole paternal genomes from biparental zygote into distinct cell linages as "heterogoneic cell division", which was proven in the bovine exam. That may be a reasonable mechanism in our case as well, explaining why androgenetic $45, X$ forms. With regard to the mosaicism of biparental $46, X$, isoXq/45X, it would be due to an oocyte carrying only the $\mathrm{q}$ arm on $\mathrm{X}$ chromosome and a failed centromere division during metaphase II, according to David F. Callen's proposed mechanism [12].

The limitations of these genetic evaluations are the following: (1) During cytogenetic cultures, some cells with uniparental genome or aneuploidy may be hard to grow, thus we cannot identify the definite genotypes of all cell lines; (2) Microscopically, there are still a lot of normal villi around the dysplastic cysts, so the sample is mixed and contaminated. If available, microdissection should be considered to isolate the truly dysplastic cells; (3) In our study, only cord blood and amniotic fluid of the fetus were taken for evaluation, which is not sufficiently representative of all germ layers. In addition, only one specimen on each site of the placenta was collected, thus the within-group variation could not be addressed.

\section{Conclusions}

This is a unique case, which presented PMD combined with mosaic biparental 46, $X$, iso $(X q)$ and androgenetic $45, X$. The highest ratio of cells with androgenetic $(45, X)$ was detected in the dysplastic placenta.

Author Contributions: Conceptualization, Y.-L.C. and C.-C.H.; Methodology, S.-D.C., C.-H.L., T.M.K., S.-H.U., Y.-H.C. and M.-C.W.; Writing-Original draft preparation, Y.-L.C., C.-C.H. and S.-D.C.; Writing-Review and editing, Y.-L.C., C.-H.L., C.-C.H., T.-M.K., S.-H.U., Y.-H.C. and S.-D.C. All authors have read and agreed to the published version of the manuscript.

Funding: This research did not receive any specific grant from funding agencies in the public, commercial, or not-for-profit sectors.

Institutional Review Board Statement: This study was approved by the Institute Review Board of Chang Gung Memorial Hospital (202101965B0).

Informed Consent Statement: Informed consent and consent for publication were obtained from the patient's parents. A copy of the written consent is available for review by the editor of this journal.

Data Availability Statement: The datasets obtained and/or analyzed during the current study are available from the corresponding author on reasonable request.

Conflicts of Interest: The authors declare no conflict of interest.

\section{References}

1. Hodgson, A.; Dube, V.; Strickland, S.; Kolomietz, E.; Noor, A.; Akbari, A.; Colgan, T.; Turashvili, G. Androgenetic/Biparental Mosaic/Chimeric Conceptions with a Molar Component: A Diagnostic and Clinical Challenge. Int. J. Gynecol. Pathol. 2021, 40, 510-517. [CrossRef] [PubMed]

2. Nayeri, U.A.; West, A.B.; Grossetta Nardini, H.K.; Copel, J.A.; Sfakianaki, A.K. Systematic review of sonographic findings of placental mesenchymal dysplasia and subsequent pregnancy outcome. Ultrasound Obstet. Gynecol. 2013, 41, 366-374. [CrossRef] [PubMed]

3. Kaiser-Rogers, K.A.; McFadden, D.E.; Livasy, C.A.; Dansereau, J.; Jiang, R.; Knops, J.F.; Lefebvre, L.; Rao, K.W.; Robinson, W.P. Androgenetic/biparental mosaicism causes placental mesenchymal dysplasia. J. Med. Genet. 2006, 43, 187-192. [CrossRef] [PubMed]

4. Benn, P.A.; Clive, J.M.; Collins, R. Medians for second-trimester maternal serum alpha-fetoprotein, human chorionic gonadotropin, and unconjugated estriol; differences between races or ethnic groups. Clin. Chem. 1997, 43, 333-337. [CrossRef] [PubMed]

5. Hwa, H.L.; Chang, Y.Y.; Lee, J.C.; Yin, H.Y.; Chen, Y.H.; Tseng, L.H.; Su, Y.N.; Ko, T.M. Thirteen X-chromosomal short tandem repeat loci multiplex data from Taiwanese. Int. J. Leg. Med. 2009, 123, 263-269. [CrossRef] [PubMed]

6. Allias, F.; Lebreton, F.; Collardeau-Frachon, S.; Azziza, J.; Pasquier, C.J.; Arcin-Thoury, F.; Patrier, S.; Devouassoux-Shisheboran, M. Immunohistochemical expression of p57 in placental vascular proliferative disorders of preterm and term placentas. Fetal Pediatr. Pathol. 2009, 28, 9-23. [CrossRef] [PubMed] 
7. Zeng, X.; Chen, M.F.; Bureau, Y.A.; Brown, R. Placental mesenchymal dysplasia and an estimation of the population incidence. Acta Obstet. Gynecol. Scand. 2012, 91, 754-757. [CrossRef] [PubMed]

8. Arizawa, M.; Nakayama, M. Suspected involvement of the X chromosome in placental mesenchymal dysplasia. Congenit. Anom. (Kyoto) 2002, 42, 309-317. [CrossRef] [PubMed]

9. Guzel Erdal, G.; Balkan, M. Turner Syndrome with Isochromosome Xq. J. Pediatr. Genet. 2020, 9, 243-245. [CrossRef] [PubMed]

10. Berkowitz, R.S.; Sandstrom, M.; Goldstein, D.P.; Driscoll, S.G. 45,X complete hydatidiform mole. Gynecol. Oncol. 1982, 14, $279-283$. [CrossRef]

11. Destouni, A.; Zamani Esteki, M.; Catteeuw, M.; Tšuiko, O.; Dimitriadou, E.; Smits, K.; Kurg, A.; Salumets, A.; Van Soom, A.; Voet, T.; et al. Zygotes segregate entire parental genomes in distinct blastomere lineages causing cleavage-stage chimerism and mixoploidy. Genome Res. 2016, 26, 567-578. [CrossRef] [PubMed]

12. Callen, D.F.; Mulley, J.C.; Baker, E.G.; Sutherland, G.R. Determining the origin of human X isochromosomes by use of DNA sequence polymorphisms and detection of an apparent $\mathrm{i}(\mathrm{Xq})$ with Xp sequences. Hum. Genet. 1987, 77, 236-240. [CrossRef] 